\title{
ALMOST AUTOMORPHIC SOLUTIONS FOR EVOLUTIONS EQUATIONS
}

\author{
Bruno de Andrade — Eder Mateus - Arlúcio Viana
}

\begin{abstract}
In this work we deal with existence and uniqueness of almost automorphic solutions for abstract semilinear differential equations using a mix of fixed point theory and extrapolation spaces theory. We apply our abstract results in the framework of transmission problems for the Bernoulli-Euler plate equation and heat conduction theory.
\end{abstract}

\section{Introduction}

In recent years, the theory of almost automorphic functions has been developed extensively and consequently there has been a considerable interest in the existence of almost automorphic solutions of various kinds of evolution equations, see for instance [4], [6], [8], [10] and the references therein. In this work, we study existence and uniqueness of almost automorphic mild solutions for a class of abstract differential equations described in the form

$$
u^{\prime}(t)=A u(t)+f(t, u(t)), \quad t \in \mathbb{R},
$$

where $A$ is an unbounded linear operator, assumed to be Hille-Yosida of negative type, with domain $D(A)$ not necessarily dense on some Banach space $X$, $f: \mathbb{R} \times X_{0} \rightarrow X$ is a continuous function and $X_{0}=\overline{D(A)}$.

2010 Mathematics Subject Classification. Primary 39B05, 35B99; Secondary 35R15, $35 \mathrm{~K} 05$.

Key words and phrases. Functional differential equations, almost automorphic solutions, transmission problems, heat equation.

Bruno de Andrade is partially supported by CNPQ/Brazil under Grant 100994/2011-3. 
It is well known that if the domain of the linear operator $A$ is non-dense, the classical theory of semigroups cannot be applied directly to treat the equation (1.1). Indeed, the Hille-Yosida theorem gives necessary and sufficient conditions for a linear operator to generate a $C_{0}$-semigroup, among these conditions there is the density of the domain of the operator. On the other hand, there are a great variety of semilinear differential equations with a linear part dominated by a nondensely defined operator. Such situations arise, for example, from restrictions made on the space where the equation is considered (e.g. periodic continuous functions, Hölder continuous functions) and from boundary conditions (e.g. the set of continuous functions with null value on the boundary is non-dense in the space of continuous functions).

However, in [14] the authors show that if the usual Hille-Yosida conditions are satisfied, but without the assumption that $D(A)$ be dense in $X$, then existence and uniqueness results for the problem (1.1) can be obtained which are even more general than those known when $D(A)$ is dense in $X$. The main tool used in [14] was the abstract extrapolation theory. First attempts to build this theory where made by Da Prato and Grisvard [13] and Nagel [9] and used from various purposes.

The main results of this work are contained in Sections 3 and 4. Basically, they says that if the non-linear term in (1.1) is an almost automorphic function, in some sense, and is a locally or globally Lipschitz continuous function, then the equation (1.1) has a unique almost automorphic mild solution. As typical applications of our abstract results we consider, e.g. the transmission problem for the Bernoulli-Euler plate equation. Precisely, let $\Omega_{1} \subset \Omega \subset \mathbb{R}^{n}, n \geq 2$, be strictly convex, bounded domains with smooth boundaries $\Gamma_{1}=\partial \Omega_{1}$ and $\Gamma=\partial \Omega$ with $\Gamma_{1} \cap \Gamma=\emptyset$. Then $\mathcal{O}=\Omega \backslash \Omega_{1}$ is a bounded, connected domain with boundary $\partial \mathcal{O}=\Gamma_{1} \cup \Gamma$. We are going to study the following mixed boundary value problem

$$
\begin{cases}\left(\partial_{t}^{2}+c^{2} \Delta^{2}\right) u_{1}(x, t)=b(t) f\left(u_{1}(x, t)\right) & \text { in } \Omega_{1} \times \mathbb{R}, \\ \left(\partial_{t}^{2}+\Delta^{2}\right) u_{2}(x, t)=b(t) f\left(u_{2}(x, t)\right) & \text { in } \mathcal{O} \times \mathbb{R}, \\ \left.u_{1}\right|_{\Gamma_{1}}=\left.u_{2}\right|_{\Gamma_{1}},\left.\quad \partial_{\nu} u_{1}\right|_{\Gamma_{1}}=\left.\partial_{\nu} u_{2}\right|_{\Gamma_{1}}, & \\ \left.c \Delta u_{1}\right|_{\Gamma_{1}}=\left.\Delta u_{2}\right|_{\Gamma_{1}},\left.\quad c \partial_{\nu} \Delta u_{1}\right|_{\Gamma_{1}}=\left.\partial_{\nu} \Delta u_{2}\right|_{\Gamma_{1}}, & \\ \left.u_{2}\right|_{\Gamma}=0,\left.\quad \Delta u_{2}\right|_{\Gamma}=-\left.a \partial_{\nu} \partial_{t} u_{2}\right|_{\Gamma}, & \end{cases}
$$

where $c>1$ is a constant, $\nu$ denotes the inner unit normal to the boundary, $a$ is a non-negative function on $\Gamma, b: \mathbb{R} \rightarrow \mathbb{R}$ and $f: \mathbb{R} \rightarrow \mathbb{R}$ are real functions that satisfies some additional conditions. Under natural assumptions on non-linear term of (1.2), see Example 3.7, we show that this problem has a unique almost automorphic mild solution. 
This work is organized as follows. In Section 2, we collect results and standard literature of almost automorphic functions. In particular we recall a result of composition for almost autmorphic functions (see Lemma 2.4) which is very important in our investigations. In Section 3, we study existence and uniqueness of almost autmorphic mild solutions for the equation (1.1) with almost autmorphic conditions. In Section 4, we ensure sufficient conditions for existence and uniqueness of almost autmorphic solutions to (1.1) with Stepanov almost autmorphic conditions.

Acknowledgements. This paper was initiated while the first author was visiting the Universidade Federal de Sergipe (Sergipe, Brasil) during May 2011. B. de Andrade would like to thank the DMAI-UFS for they kind invitation and hospitality.

\section{Preliminaries}

Before stating the precise problems of concern in this paper, let us introduce the basic results and notations we will be considering. Consider $(X,\|\cdot\|)$ and $(Y,\|\cdot\|)$ Banach spaces. In this work $B C(\mathbb{R} ; X)$ denotes the space consisting of the continuous and bounded functions from $\mathbb{R}$ into $X$ endowed with the norm of the uniform convergence which is denoted for $\|\cdot\|_{\infty}$. Furthermore, the notation $\mathcal{L}(X, Y)$ stands for the space of bounded linear operators from $X$ into $Y$ endowed with the uniform operator topology, and we abbreviate to $\mathcal{L}(X)$ whenever $X=Y$.

2.1. Almost automorphic functions. We begin recalling the notion of almost automorphic function $\left({ }^{1}\right)$.

Definition 2.1. A continuous function $f: \mathbb{R} \mapsto X$ is called almost automorphic if for every sequence of real numbers $\left(s_{n}^{\prime}\right)_{n \in \mathbb{N}}$ there exists a subsequence $\left(s_{n}\right)_{n \in \mathbb{N}} \subset\left(s_{n}^{\prime}\right)_{n \in \mathbb{N}}$ such that

$$
\lim _{n, m \rightarrow \infty}\left\|f\left(t+s_{n}-s_{m}\right)-f(t)\right\|=0 .
$$

Example 2.2. Concrete examples of almost automorphic functions are given by the functions

$$
a(t)=\cos \left(\frac{1}{\cos (t)+\cos (\sqrt{2} t)}\right) \quad \text { and } \quad b(t)=\sin \left(\frac{1}{\sin (t)+\sin (\sqrt{2} t)}\right),
$$

for $t \in \mathbb{R}$.

Definition 2.3. Let $X$ and $Y$ be two Banach spaces. A continuous function $f: \mathbb{R} \times Y \mapsto X$ is said to be almost automorphic if $f(t, x)$ is almost automorphic in $t \in \mathbb{R}$ uniformly for all $x \in K$, where $K$ is any bounded subset of $Y$.

$\left({ }^{1}\right)$ This definition is due to Bochner (see [3]). 


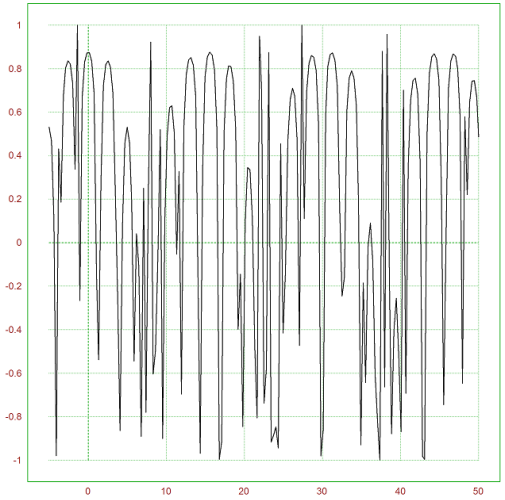

(a) $\cos \left(\frac{1}{\cos (t)+\cos (\sqrt{2} t)}\right)$

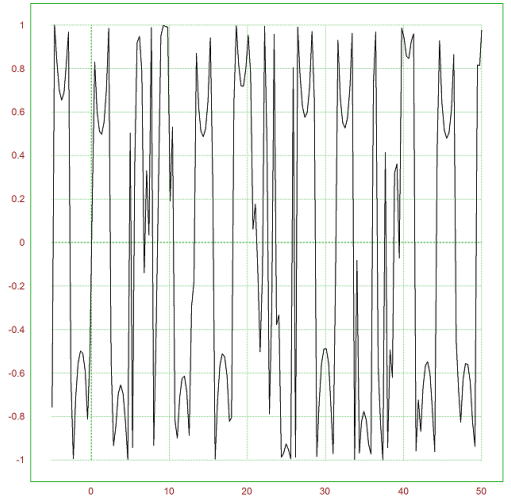

(b) $\sin \left(\frac{1}{\sin (t)+\sin (\sqrt{2} t)}\right)$

Figure 1

In this work we use the notation $\mathrm{AA}(X)$ to represent the subset of $\mathrm{BC}(\mathbb{R} ; X)$ formed by the almost automorphic functions. We observe that $\left(\mathrm{AA}(X),\|\cdot\|_{\infty}\right)$ is a Banach space. Furthermore, if $f \in \mathrm{AA}(X)$ then the set $\{f(t): t \in \mathbb{R}\}$ is a relatively compact subset of $X$. Similarly, we set $\mathrm{AA}(Y ; X)$ to represent the set of all functions almost automorphic in $t$ uniformly for $x \in Y$.

The following result is standard in the theory of almost automorphic functions.

Lemma 2.4 [7]. If $f: \mathbb{R} \times Y \mapsto X$ is almost automorphic, and $h \in \mathrm{AA}(Y)$, and assume that $f(t, \cdot)$ is uniformly continuous on each bounded subset $K \subset Y$ uniformly for $t \in \mathbb{R}$, that is for any $\varepsilon>0$, there exists $\delta>0$ such that $x, y \in K$ and $\|x-y\|<\delta$ imply that $\|f(t, x)-f(t, y)\|<\varepsilon$ for all $t \in \mathbb{R}$, then the function $f(\cdot, h(\cdot)) \in \mathrm{AA}(X)$.

2.2. Stepanov almost automorphic functions. Let $(X,\|\cdot\|),(Y,\|\cdot\|)$ be Banach spaces. We remember the concept of Stepanov almost automorphic functions.

Definition 2.5. The space $\mathrm{BS}^{p}(X)$ of all Stepanov functions, with the exponent $p$, consists of all measurable functions $f$ on $\mathbb{R}$ with values in $X$ such that

$$
\|f\|_{\mathrm{S}^{p}}:=\sup _{t \in \mathbb{R}}\left(\int_{t}^{t+1}\|f(\tau)\|^{p} d \tau\right)^{1 / p}<+\infty .
$$

We observe that, whenever $p \geq q \geq 1, L^{p}(\mathbb{R}, X) \subseteq \mathrm{BS}^{p}(X) \subseteq L_{\text {loc }}^{p}(\mathbb{R}, X)$ and $\operatorname{BS}^{p}(X) \subseteq \operatorname{BS}^{q}(X)$. Furthermore, for each $p \geq 1$, we have the following continuous inclusion

$$
\left(\mathrm{BC}(X),\|\cdot\|_{\infty}\right) \hookrightarrow\left(\mathrm{BS}^{p}(X),\|\cdot\|_{\mathrm{S}^{p}}\right) .
$$


REMARK 2.6. In the above definition, we can replace the norm $\|\cdot\|_{S^{p}}$ by the norm obtained varying the length of the integration interval as

$$
\|f\|=\sup _{t \in \mathbb{R}}\left(\frac{1}{l} \int_{t}^{t+l}\|f(\tau)\|^{p} d \tau\right)^{1 / p},
$$

where $l>0$ and we obtain a new norm equivalent to the old one.

Definition 2.7. A function $f \in \mathrm{BS}^{p}(X)$ is called Stepanov almost automorphic if for every sequence of real numbers $\left(s_{n}^{\prime}\right)_{n \in \mathbb{N}}$ there are a subsequence $\left(s_{n}\right)_{n \in \mathbb{N}} \subset\left(s_{n}^{\prime}\right)_{n \in \mathbb{N}}$ and a function $g \in L^{p}(\mathbb{R}, X)$ such that

$$
\left(\int_{0}^{1}\left\|f\left(t+s_{n}+s\right)-g(t+s)\right\|^{p} d s\right)^{1 / p} \rightarrow 0
$$

and

$$
\left(\int_{0}^{1}\left\|g\left(t-s_{n}+s\right)-f(t+s)\right\|^{p} d s\right)^{1 / p} \rightarrow 0
$$

as $n \rightarrow \infty$ pointwise on $\mathbb{R}$.

We denote the set of all Stepanov almost automorphic functions by $\operatorname{AS}^{p}(X)$. For $p \geq q \geq 1$, we have that

$$
\operatorname{AA}(X) \subseteq \operatorname{AS}^{p}(X) \subseteq \operatorname{AS}^{q}(X)
$$

EXAmple 2.8 [11]. Let $\left(x_{n}\right)_{n \in \mathbb{N}} \subset X$ be an almost automorphic sequence and consider $\epsilon \in(0,1 / 2)$. Define the function

$$
f(t)= \begin{cases}x_{n} & \text { for } t \in(n-\varepsilon, n+\varepsilon), \\ 0 & \text { otherwise }\end{cases}
$$

Then $f \in \operatorname{AS}^{p}(X)$ for every $p \geq 1$ and $f \notin \mathrm{AA}(X)$.

Definition 2.9. A function $f: \mathbb{R} \times Y \rightarrow X$ is called Stepanov almost automorphic in $t \in \mathbb{R}$ for $x \in Y$, if $f(\cdot, u) \in \operatorname{AS}^{p}(X)$ for each $u \in Y$.

We denote the set of all Stepanov almost automorphic functions $f: \mathbb{R} \times Y \rightarrow X$ by $\operatorname{AS}^{p}(Y, X)$. We close this subsection with a composition result. The proof is a standard application of the Minkowski's inequality.

Lemma 2.10. Let $f \in \operatorname{AS}^{p}(Y, X)$ and suppose that there is $L>0$ such that

$$
\|f(t, x)-f(t, y)\| \leq L\|x-y\|,
$$

for all $t \in \mathbb{R}$ and for all $x, y \in Y$. If $\phi \in \mathrm{AS}^{p}(Y)$ then the function $f(\cdot, \phi(\cdot)) \in$ $\operatorname{AS}^{p}(X)$. 
2.3. Hille-Yosida operators. We recall some basic properties of extrapolation spaces for Hille-Yosida operators which are a natural tool in our setting.

Definition 2.11. Let $X$ be a Banach space and $A$ be a linear operator with domain $D(A)$. One says that $(A, D(A))$ is a Hille-Yosida operator on $\mathrm{X}$ if there exist $\omega \in \mathbb{R}$ and a positive constant $M \geq 1$ such that $(\omega, \infty) \subset \rho(A)$ and

$$
\sup \left\{(\lambda-\omega)^{n}\left\|(\lambda-A)^{-n}\right\|: n \in \mathbb{N}, \lambda>\omega\right\} \leq M .
$$

The infinimum of such $\omega$ is called the type of $A$. If the constant $\omega$ can be chosen smaller than zero, $A$ is called of negative type.

Let $(A, D(A))$ be a Hille-Yosida operator on $\mathrm{X}$ and $X_{0}=\overline{D(A)}$. Let $A_{0}: D\left(A_{0}\right) \subset X_{0} \rightarrow X_{0}$ be the realization of $A$ on

$$
D\left(A_{0}\right)=\left\{x \in D(A): A x \in X_{0}\right\} .
$$

We have the following result.

Lemma 2.12 [5]. The operator $A_{0}$ is the infinitesimal generator of a $C_{0}$ semigroup $\left(T_{0}(t)\right)_{t \geq 0}$ on $X_{0}$ with $\left\|T_{0}(t)\right\| \leq M e^{\omega t}$ for $t \geq 0$. Moreover, $\rho(A) \subset$ $\rho\left(A_{0}\right)$ and $R\left(\lambda, A_{0}\right)=\left.R(\lambda, A)\right|_{X_{0}}$, for $\lambda \in \rho(A)$.

In this work we assume that $(A, D(A))$ is a Hille-Yosida operator of negative type on $\mathrm{X}$. This implies that $0 \in \rho(A)$, i.e. $A^{-1} \in \mathcal{L}(X)$. We remark that the expression $\|x\|_{-1}=\left\|A_{0}^{-1} x\right\|$ define a norm on $X_{0}$.

Definition 2.13. The completion of $\left(X_{0},\|\cdot\|_{-1}\right)$, denoted by $X_{-1}$, is called the extrapolation space of $X_{0}$ associated with $A_{0}$.

We note that $X$ is an intermediary space between $X_{0}$ and $X_{-1}$ and (see [9])

$$
X_{0} \hookrightarrow X \hookrightarrow X_{-1} .
$$

Since $A_{0}^{-1} T_{0}(t)=T_{0}(t) A_{0}^{-1}$, we have that $\left\|T_{0}(t) x\right\|_{-1} \leq\left\|T_{0}(t)\right\|_{\mathcal{L}\left(X_{0}\right)}\|x\|_{-1}$ which implies that $T_{0}(t)$ has a unique bounded linear extension $T_{-1}(t)$ to $X_{-1}$. The operator family $\left(T_{-1}(t)\right)_{t \geq 0}$ is a $C_{0}$-semigroup on $X_{-1}$, called the extrapolated semigroup of $\left(T_{0}(t)\right)_{t \geq 0}$. In the sequel, $\left(A_{-1}, D\left(A_{-1}\right)\right)$ is the generator of $\left(T_{-1}(t)\right)_{t \geq 0}$.

LEMma 2.14 [9]. Under the previous conditions, the following properties are verified.

(a) $D\left(A_{-1}\right)=X_{0}$ and $\left\|T_{-1}(t)\right\|_{\mathcal{L}\left(X_{-1}\right)}=\left\|T_{0}(t)\right\|_{\mathcal{L}\left(X_{0}\right)}$ for every $t \geq 0$.

(b) The operator $A_{-1}: X_{0} \rightarrow X_{-1}$ is the unique continuous extension of $A_{0}: D\left(A_{0}\right) \subset\left(X_{0},\|\cdot\|\right) \rightarrow\left(X_{-1},\|\cdot\|_{-1}\right)$ and $\lambda-A_{-1}$ is an isometry from $\left(X_{0},\|\cdot\|\right)$ into $\left(X_{-1},\|\cdot\|_{-1}\right)$.

(c) If $\lambda \in \rho\left(A_{0}\right)$, then $\left(\lambda-A_{-1}\right)^{-1}$ exists and $\left(\lambda-A_{-1}\right)^{-1} \in \mathcal{L}\left(X_{-1}\right)$. In particular, $\lambda \in \rho\left(A_{-1}\right)$ and $\left.R\left(\lambda, A_{-1}\right)\right|_{X_{0}}=R\left(\lambda, A_{0}\right)$. 
(d) The space $X_{0}=\overline{D(A)}$ is dense in $\left(X_{-1},\|\cdot\|_{-1}\right)$. Thus, the extrapolation space $X_{-1}$ is also the completion of $\left(X,\|\cdot\|_{-1}\right)$ and $X \hookrightarrow X_{-1}$. Moreover, $A_{-1}$ is an extension of $A$ to $X_{-1}$. In particular, if $\lambda \in \rho(A)$, then $\left.R\left(\lambda, A_{-1}\right)\right|_{X}=R(\lambda, A)$ and $R\left(\lambda, A_{-1}\right) X=D(A)$.

Lemma 2.15 [1]. Let $f \in \mathrm{BC}(\mathbb{R} ; X)$. Then the following properties are valid.

(a) $T_{-1} * f(t)=\int_{-\infty}^{t} T_{-1}(t-s) f(s) d s \in X_{0}$, for every $t \in \mathbb{R}$.

(b) $\left\|T_{-1} * f(t)\right\| \leq C e^{w t} \int_{-\infty}^{t} e^{-w s}\|f(s)\| d s$ where $C>0$ is independent of $t$ and $f$.

(c) The linear operator $\Lambda: \mathrm{BC}(\mathbb{R}, X) \rightarrow \mathrm{BC}\left(\mathbb{R}, X_{0}\right)$ defined by $\Lambda(f)(t)=$ $T_{-1} * f(t)$ is continuous.

(d) $\lim _{t \rightarrow 0}\left\|T_{-1} * f(t)-\int_{-\infty}^{0} T_{-1}(-s) f(s) d s\right\|=0$, for every $t \in \mathbb{R}$.

(e) $x(t)=T_{-1} * f(t)$ is the unique bounded mild solution in $X_{0}$ of $x^{\prime}(t)=$ $A x(t)+f(t), t \in \mathbb{R}$.

REMARK 2.16. For the rest of this work we assume that $(A, D(A))$ is a HilleYosida operator of negative type. Therefore, there are constants $M \geq 1$ and $\omega<0$ such that

$$
\left\|T_{-1}(t)\right\| \leq M e^{\omega t}, \quad t \geq 0,
$$

where $\left(T_{-1}(t)\right)_{t \geq 0}$ is the extrapolated semigroup associated to $\left(T_{0}(t)\right)_{t \geq 0}$.

\section{Almost automorphic solutions} with almost automorphic conditions

The next result assure the regularity of the covolution of the semigroup $\left(T_{-1}(t)\right)_{t \geq 0}$ with almost automorphic functions. This result will be very useful in our approach of the equation (1.1).

Lemma 3.1. If $u \in \mathrm{AA}\left(X_{0}\right)$, then $z: \mathbb{R} \rightarrow X$, defined by

$$
z(t):=\int_{-\infty}^{t} T_{-1}(t-s) u(s) d s
$$

is in $\mathrm{AA}\left(X_{0}\right)$.

Proof. From Lemma 2.15, we have that $z(t) \in X_{0}$. Since $u \in \operatorname{AA}\left(X_{0}\right)$, there is a sequence $\left(s_{n}\right) \subset \mathbb{R}$ and $g: \mathbb{R} \rightarrow X_{0}$ such that $g(t)=\lim _{n \rightarrow \infty} u\left(t+s_{n}\right)$ uniformly for $t \in \mathbb{R}$. Then,

$$
z\left(t+s_{n}\right)=\int_{-\infty}^{t+s_{n}} T_{-1}\left(t+s_{n}-s\right) u(s) d s=\int_{-\infty}^{t} T_{-1}(t-s) u\left(s+s_{n}\right) d s .
$$


The Dominated Convergence Theorem yields

$$
z\left(t+s_{n}\right) \rightarrow \int_{-\infty}^{t} T_{-1}(t-s) g(s) d s, \quad \text { as } n \rightarrow \infty .
$$

A similar computation shows that

$\int_{-\infty}^{t-s_{n}} T_{-1}\left(t-s_{n}-s\right) g(s) d s=\int_{-\infty}^{t} T_{-1}(t-s) g\left(s-s_{n}\right) d s \rightarrow z(t), \quad$ as $n \rightarrow \infty$, and this conclude the proof.

REMARK 3.2. An immediate consequence of Lemma 3.1 is the existence of almost automorphic mild solution for the nonhomogeneous linear peoblem

$$
u^{\prime}(t)=A u(t)+f(t), \quad t \in \mathbb{R},
$$

where $A$ is a Hille-Yosida operator of negative type on a Banach space $X$ and $f \in \operatorname{AA}\left(X_{0}\right)$.

Theorem 3.3. Let $f \in \mathrm{AA}\left(X_{0}, X\right)$ as in Lemma 2.4. Assume that there exists a function $L \in L_{\mathrm{loc}}^{1}(\mathbb{R} ;[0, \infty))$ such that

$$
\|f(t, x)-f(t, y)\| \leq L(t)\|x-y\|, \quad t \in \mathbb{R}, x, y \in X_{0} .
$$

Let

$$
\theta(t)=\int_{-\infty}^{t} e^{\omega(t-s)} L(s) d s, \quad t \in \mathbb{R} .
$$

Suppose that there is a positive constant $K<1$ such that $M \theta(t)<K$, for all $t \in \mathbb{R}$. Then the equation (1.1) has a unique mild solution in $\mathrm{AA}\left(X_{0}\right)$.

Proof. We define the operator $\Lambda$ on the space $\mathrm{AA}\left(X_{0}\right)$ by

$$
\Lambda u(t)=\int_{-\infty}^{t} T_{-1}(t-s) f(s, u(s)) d s .
$$

It follows from Lemma 2.4 that $f(\cdot, u(\cdot)) \in \mathrm{AA}(X)$ for every $u \in \mathrm{AA}\left(X_{0}\right)$. Furthermore, by Lemma 3.1 we have that the operator $\Lambda: \mathrm{AA}\left(X_{0}\right) \rightarrow \mathrm{AA}\left(X_{0}\right)$ is well defined. We next proof that $\Lambda$ is a $K$-contraction. In fact, if $u, v \in \mathrm{AA}\left(X_{0}\right)$ we have that

$$
\begin{aligned}
\|\Lambda u(t)-\Lambda v(t)\| & \leq \int_{-\infty}^{t} M e^{\omega(t-s)} L(s)\|u(s)-v(s)\| d s \\
& \leq M \theta(t)\|u-v\| \leq K\|u-v\|_{\infty} .
\end{aligned}
$$

Hence, by the Banach's fixed point theorem we conclude the proof.

The next results are immediate consequences of Theorem 3.3. 
Corollary 3.4. Let $f \in \mathrm{AA}\left(X_{0}, X\right)$ and suppose that $f$ satisfies the Lipschitz condition (3.2) with $L$ a bounded continuous function. Let

$$
\theta(t)=\int_{-\infty}^{t} e^{\omega(t-s)} L(s) d s, \quad t \in \mathbb{R} .
$$

Suppose that there is a positive constant $K<1$ such that $M \theta(t)<K$, for all $t \in \mathbb{R}$. Then the equation (1.1) has a unique mild solution in $\mathrm{AA}\left(X_{0}\right)$.

EXAMPLE 3.5. We consider a simple application of our abstract results. Let $a \in \mathrm{AA}(\mathbb{R})$ and consider the partial differential equation

$$
\begin{cases}u_{t}=u_{x x}-u+a \sin (u) & \text { in } \mathbb{R} \times[0, \pi], \\ u=0 & \text { on } \mathbb{R} \times\{0, \pi\} .\end{cases}
$$

Let $X=C([0, \pi] ; \mathbb{R})$ and define the operator $A$ on $X$ by $A u=u^{\prime \prime}-u$, with domain $D(A)=\left\{u \in X: u^{\prime \prime} \in X, u(0)=u(\pi)=0\right\}$.

It is well known that $A$ is a Hille-Yosida operator of type -1 with domain non-dense. Equation (3.4) can be rewritten as an abstract system of the form (1.1), where $u(t)(s)=u(t, s)$,

$$
f(t, \phi)(x)=a(t) \sin (\phi(x)),
$$

for all $\phi \in X, t \in \mathbb{R}$ and $x \in[0, \pi]$. If we assume that $M\|a\|_{\infty}<|\omega|$, then the equation (3.4) has a unique almost automorphic mild solution.

Corollary 3.6. Let $f \in \mathrm{AA}\left(X_{0}, X\right)$ and suppose that $f$ satisfies the Lipschitz condition

$$
\|f(t, x)-f(t, y)\| \leq K\|x-y\| \quad \text { for all } x, y \in X_{0} \text { and } t \in \mathbb{R} .
$$

If $K$ is small enough, then the equation (1.1) has a unique mild solution in $\operatorname{AA}\left(X_{0}\right)$.

ExAmple 3.7. Consider the transmission problem for the Bernoulli-Euler plate equation (1.2). We suppose that there is a constant $a_{0}>0$ such that $a_{0} \leq a$ on $\Gamma$. Furthermore, we assume that $b \in \mathrm{AA}(\mathbb{R})$. Consider the function

$$
\alpha(x)= \begin{cases}c & \text { in } \Omega_{1} \\ 1 & \text { in } \mathcal{O}\end{cases}
$$

To treat the problem (1.2) we will follow some ideas of [2]. To this end, introduce the Hilbert space $\mathcal{H}=V \times H$, where $H=L^{2}\left(\Omega, \alpha(x)^{-1} d x\right)$ and the space $V$ is defined as follows. On the Hilbert space $H$ consider the operator $G$ defined by

$$
G\left(\begin{array}{l}
u_{1} \\
u_{2}
\end{array}\right)=\left(\begin{array}{c}
-c \Delta u_{1} \\
-\Delta u_{2}
\end{array}\right), \quad \text { for all }\left(\begin{array}{l}
u_{1} \\
u_{2}
\end{array}\right) \in D(G),
$$


with domain

$$
\begin{aligned}
D(G)=\left\{\left(u_{1}, u_{2}\right) \in H=L^{2}\left(\Omega_{1}, c^{-1} d x\right) \oplus L^{2}(\mathcal{O}): u_{1} \in H^{2}\left(\Omega_{1}\right),\right. \\
\\
\left.\quad u_{2} \in H^{2}(\mathcal{O}),\left.u_{2}\right|_{\Gamma}=0,\left.u_{1}\right|_{\Gamma_{1}}=\left.u_{2}\right|_{\Gamma_{1}},\left.\partial_{\nu} u_{1}\right|_{\Gamma_{1}}=\left.\partial_{\nu} u_{2}\right|_{\Gamma_{1}}\right\} .
\end{aligned}
$$

The operator $G$ is a strictly positive self-adjoint one with a compact resolvent. Set $V=D(G)$ with norm $\|\cdot\|_{V}=\|G \cdot\|_{H}$.

We can rewrite (1.2) as follows. Define the operator $A: D(A) \subset \mathcal{H} \rightarrow \mathcal{H}$ by

$$
A\left(\begin{array}{c}
u \\
u
\end{array}\right)=-i\left(\begin{array}{c}
v \\
-\alpha^{2} \Delta^{2} u
\end{array}\right), \quad \text { for all }\left(\begin{array}{c}
u \\
v
\end{array}\right) \in D(A),
$$

where

$$
\begin{aligned}
& D(A)=\left\{(u, v) \in \mathcal{H}:\left(v, \Delta^{2} u\right) \in \mathcal{H},\left.u\right|_{\Gamma}=0,\left.\Delta u\right|_{\Gamma}=-\left.a \partial_{\nu} v\right|_{\Gamma}\right. \\
& \left.\left.u\right|_{\Gamma_{1}}=\left.v\right|_{\Gamma_{1}},\left.\partial_{\nu} u\right|_{\Gamma_{1}}=\left.\partial_{\nu} v\right|_{\Gamma_{1}},\left.c \Delta u\right|_{\Gamma_{1}}=\left.\Delta v\right|_{\Gamma_{1}},\left.c \partial_{\nu} \Delta u\right|_{\Gamma_{1}}=\left.\partial_{\nu} \Delta v\right|_{\Gamma_{1}}\right\} .
\end{aligned}
$$

Using Green's formula we can see that

$$
\operatorname{Im}\left\langle A\left(\begin{array}{l}
u \\
u
\end{array}\right),\left(\begin{array}{l}
u \\
u
\end{array}\right)\right\rangle_{\mathcal{H}}=\int_{\Gamma} a\left|\partial_{\nu} v\right|^{2} d \Gamma \geq 0, \quad \text { for all }\left(\begin{array}{c}
u \\
u
\end{array}\right) \in D(A) .
$$

This implies that $A$ generates a $C_{0}$-semigroup $(T(t))_{t \geq 0}$ (e.g. see Theorems 4.3 and 4.6 from [12, p.14-15]). On the other hand, follows from [2] that the resolvent set $\rho(A)$ is a discrete set of eigenvalues of $A$. Moreover, $A$ has no eigenvalue on the real axis. Consequently, there are constants $M \geq 1$ and $\omega<0$ such that $\|T(t)\| \leq M e^{\omega t}, t \geq 0$. Finally, we will assume that the function $f: \mathbb{R} \rightarrow \mathbb{R}$ is a globally Lipschitz continuous function with constant $K>0$. If $K$ is small enough, then the problem (1.2) has a unique almost automorphic mild solution.

We now move on to the problem of locally Lipschitz perturbations for equation (1.1). In this sense, the following theorem is the main result of this section.

REMARK 3.8. In the following result we will use the notion of locally bounded function, that is, we consider a function $L: X_{0} \times X_{0} \rightarrow[0, \infty)$ such that for every $r \geq 0$ there is a constant $k(r) \geq 0$ such that $L(x, y) \leq k(r)$, for all $x, y \in X_{0}$ with $\|x\| \leq r$ and $\|y\| \leq r$.

Theorem 3.9. Consider $f \in \mathrm{AA}\left(X_{0}, X\right)$ and let $L: X_{0} \times X_{0} \rightarrow[0, \infty)$ be a locally bounded function such that

$$
\|f(t, x)-f(t, y)\| \leq L(x, y)\left(1+\|x\|^{l-1}+\|y\|^{l-1}\right)\|x-y\|, \quad \text { for all } t \in \mathbb{R}
$$

with $l>1$. Suppose that there is $R>0$ such that

$$
\left(K(R)+\frac{\|f(\cdot, 0)\|_{\infty}}{R}\right) \frac{M}{|\omega|}<1, \quad \text { where } K(R):=\frac{k(R)\left(R+2 R^{l}\right)}{R},
$$

with $k(R)$ as in Remark 3.8. Then, the equation (1.1) has a unique mild solution in $\mathrm{AA}\left(X_{0}\right)$. 
Proof. Define the operator $\Lambda$ by expression (3.3). Consider $R>0$ such that $\left(R K(R)+\|f(\cdot, 0)\|_{\infty}\right) M /|\omega|<R$.

Let $B_{R}$ be the closed ball $B_{R}=\left\{u \in \mathrm{AA}\left(X_{0}\right):\|u\|_{\infty} \leq R\right\} \subset \mathrm{AA}\left(X_{0}\right)$. We observe that, if $u \in B_{R}$, then

$$
\begin{aligned}
\|\Lambda u(t)\| \leq & \int_{-\infty}^{t}\left\|T_{-1}(t-s) f(s, u(s))\right\| d s \\
\leq & M \int_{-\infty}^{t} e^{\omega(t-s)} L(u(s), 0)\left(1+\|u(s)\|^{l-1}\right)\|u(s)\| d s \\
& +M\|f(\cdot, 0)\|_{\infty} \int_{-\infty}^{t} e^{\omega(t-s)} d s \\
\leq & \left(R K(R)+\|f(\cdot, 0)\|_{\infty}\right) \frac{M}{|\omega|} \leq R .
\end{aligned}
$$

Therefore, $\Lambda\left(B_{R}\right) \subset B_{R}$. It remains to show that $\Lambda$ is a contraction. But this follows from estimate

$$
\begin{aligned}
& \|\Lambda u(t)-\Lambda v(t)\| \leq \int_{-\infty}^{t}\left\|T_{-1}(t-s)(f(s, u(s))-f(s, v(s)))\right\| d s \\
& \quad \leq M \int_{-\infty}^{t} e^{\omega(t-s)} L(u(s), v(s))\left(1+\|u(s)\|^{l-1}+\|v(s)\|^{l-1}\right) d s\|u-v\|_{\infty} \\
& \quad \leq\left(\frac{M K(R)}{|\omega|}\right)\|u-v\|_{\infty}
\end{aligned}
$$

Since $M K(R) /|\omega|<1$, the assertion is a consequence of the Banach fixed point theorem and the proof is completed.

COROLlary 3.10. Let $f \in \mathrm{AA}\left(X_{0}, X\right)$ and assume that there is a constant $c \geq 0$ such that for every $x, y \in X_{0}$ we have

$$
\|f(t, x)-f(t, y)\| \leq c\left(1+\|x\|^{l-1}+\|y\|^{l-1}\right)\|x-y\|, \quad \text { for all } t \in \mathbb{R},
$$

with $l \geq 1$. If $c$ is small enough then the equation (1.1) has a unique almost automorphic mild solution.

COROLlary 3.11. Let $f \in \mathrm{AA}\left(X_{0}, X\right)$ and assume that for every $r \geq 0$ there is a constant $L(r) \geq 0$ such that for every $x, y \in X_{0}$, with $\|x\| \leq r$ and $\|y\| \leq r$, we have

$$
\|f(t, x)-f(t, y)\| \leq L(r)\|x-y\|, \quad \text { for all } t \in \mathbb{R} .
$$

If there is $R>0$ such that

$$
\left(L(R)+\frac{\|f(\cdot, 0)\|_{\infty}}{R}\right) \frac{M}{|\omega|}<1 .
$$

Then, the equation (1.1) has a unique mild solution in $\mathrm{AA}\left(X_{0}\right)$. 
Example 3.12. Consider the set $B=\left\{x \in \mathbb{R}^{n}:\|x\|<1\right\}$ and let $S^{n-1}=$ $\partial B$. We study existence and uniqueness of almost automorphic mild solutions for the nonhomogeneous equation:

$$
\begin{cases}u_{t}=\Delta u+a g(u) & \text { in } \mathbb{R} \times B, \\ u=0 & \text { on } \mathbb{R} \times S^{n-1},\end{cases}
$$

where $a \in \mathrm{AA}(\mathbb{R})$ and $g \in C^{1}(\mathbb{R}, \mathbb{R})$ satisfies the additional condition:

$$
\lim _{|s| \rightarrow \infty} \frac{\left|g^{\prime}(s)\right|}{|s|^{l-1}}=0,
$$

with $l>1$. Then for each $\eta>0$ there is $C_{\eta}>0$ such that

$$
\left|g\left(s_{1}\right)-g\left(s_{2}\right)\right| \leq\left(C_{\eta}+\eta\left|s_{1}\right|^{l-1}+\eta\left|s_{2}\right|^{l-1}\right)\left|s_{1}-s_{2}\right|, \quad \text { for all } s_{1}, s_{2} \in \mathbb{R} .
$$

To treat system (3.9), we choose the space $X=C(\bar{B} ; \mathbb{R})$ and the operator $A$ defined by $A v=\Delta v+\omega v, \omega<0$, with domain

$$
D(A)=\left\{v \in X: v=0 \text { on } S^{n-1} \text { and } \Delta v \in X\right\} .
$$

In this case, $X_{0}=C_{0}(\bar{B} ; \mathbb{R}) \neq X$ and therefore $A$ is a Hille-Yosida operator of negative type $\omega$ with non-dense domain. Its clear that (3.9) can be rewritten as an abstract system of the form

$$
u^{\prime}(t)=A u(t)+f(t, u(t)), \quad t \in \mathbb{R},
$$

where $u(t)(x)=u(t, x)$ and $f(t, \psi)(x)=a(t) f(\psi(x))-\omega \psi(x), t \in \mathbb{R}, x \in B$. Furthermore, we have that

$\left\|f\left(t, \psi_{1}\right)-f\left(t, \psi_{2}\right)\right\| \leq c\left(1+\left\|\psi_{1}\right\|^{l-1}+\left\|\psi_{2}\right\|^{l-1}\right)\left\|\psi_{1}-\psi_{2}\right\|, \quad$ for all $\psi_{1}, \psi_{2} \in X_{0}$.

Hence, if $c=c(\omega)>0$ is small enough the system (3.9) has a unique almost automorphic mild solution.

\section{Almost automorphic solutions}

\section{with Stepanov almost automorphic conditions}

This section deals with existence and uniqueness of almost automorphic mild solution to equation (1.1) with Stepanov almost automorphic conditions. We fix $q=p /(p-1), p>1$. We have the following auxiliary result:

Lemma 4.1. Let $f \in \operatorname{AS}^{p}(X) \cap C(\mathbb{R}, X)$, with $p>1$. Given $n \in \mathbb{N}$, consider

$$
v_{n}(t)=\int_{n-1}^{n} T_{-1}(\xi) f(t-\xi) d \xi
$$

Then, $v_{n} \in \mathrm{AA}\left(X_{0}\right)$, for every $n \in \mathbb{N}$. 
Proof. In fact, let $\left(s_{m}\right)_{m \in \mathbb{N}} \subset \mathbb{R}$. Since $f \in \mathrm{AS}^{p}(X)$, there are a subsequence $\left(s_{m_{k}}\right)_{k \in \mathbb{N}} \subset\left(s_{m}\right)_{m \in \mathbb{N}}$ and a function $g \in \operatorname{AS}^{p}(X)$ such that

$$
\left(\int_{0}^{1}\left\|f\left(t+s_{m_{k}}+\sigma\right)-g(t+\sigma)\right\|^{p} d \sigma\right)^{1 / p} \rightarrow 0
$$

as $k \rightarrow \infty$. Let $\widetilde{v}_{n}=\int_{n-1}^{n} T_{-1}(\xi) g(t-\xi) d \xi$. Follows from the Hölder's inequality that

$$
\begin{aligned}
\| v_{n}\left(t+s_{m_{k}}\right) & -\widetilde{v}_{n}(t)\|=\| \int_{n-1}^{n} T_{-1}(\xi)\left(f\left(t+s_{m_{k}}-\xi\right)-g(t-\xi)\right) d \xi \| \\
\leq & M \int_{n-1}^{n} e^{\omega \xi}\left\|\left(f\left(t+s_{m_{k}}-\xi\right)-g(t-\xi)\right)\right\| d \xi \\
\leq & M\left(\int_{n-1}^{n} e^{q \omega \xi} d \xi\right)^{1 / q}\left(\int_{n-1}^{n}\left\|\left(f\left(t+s_{m_{k}}-\xi\right)-g(t-\xi)\right)\right\|^{p} d \xi\right)^{1 / p} \\
& =K\left(\int_{n-1}^{n}\left\|\left(f\left(t++s_{m_{k}}-\xi\right)-g(t-\xi)\right)\right\|^{p} d \xi\right)^{1 / p},
\end{aligned}
$$

where $K=K(q, \omega, M)$ is constant. Then, (4.1) implies that

$$
\left\|v_{n}\left(t+s_{m_{k}}\right)-\widetilde{v}_{n}(t)\right\| \rightarrow 0
$$

as $k \rightarrow \infty$. Similarly, we have that

$$
\left\|\widetilde{v}_{n}\left(t-s_{m}\right)-v_{n}(t)\right\| \rightarrow 0
$$

as $k \rightarrow \infty$, and this conclude the proof.

Lemma 4.2. Let $f \in \operatorname{AS}^{p}(X) \cap C(\mathbb{R}, X)$, with $p>1$. Then the function

$$
u(t)=\int_{-\infty}^{t} T_{-1}(t-s) f(s) \in \operatorname{AA}\left(X_{0}\right) .
$$

Proof. Let $\left(v_{n}\right)_{n \in \mathbb{N}}$ be as in the Lemma 4.1. Note that

$$
v_{n}(t)=\int_{t-n}^{t-n+1} T_{-1}(t-s) f(s) d s
$$

Hence,

$$
\left\|v_{n}\right\| \leq M \int_{t-n}^{t-n+1} e^{\omega(t-s)}\|f(s)\| d s .
$$

Using the Hölder's inequality, we obtain

$$
\begin{aligned}
\left\|v_{n}\right\| & \leq M\left(\int_{t-n}^{t-n+1} e^{q \omega(t-s)} d s\right)^{1 / q}\left(\int_{t-n}^{t-n+1}\|f(s)\|^{p}\right)^{1 / p} d s \\
& \leq \frac{M}{\sqrt[q]{q|\omega|}}\left(e^{q \omega(n-1)}-e^{q \omega n}\right)^{1 / q}\|f\|_{S^{p}} \\
& \leq \frac{M e^{\omega n}}{\sqrt[q]{q|\omega|}}\left(e^{q|\omega|}-1\right)^{1 / q}\|f\|_{S^{p}} \leq \frac{M e^{\omega}}{\sqrt[q]{q|\omega|}}\left(e^{q|\omega|}+1\right)^{1 / q}\|f\|_{S^{p}} .
\end{aligned}
$$


Since the series

$$
\left(\frac{M e^{\omega}}{\sqrt[q]{q|\omega|}}\left(e^{q|\omega|}+1\right)^{1 / q}\right) \sum_{n=1}^{\infty} e^{\omega n}
$$

is convergent, follows from the Weierstrass test that $\sum_{n=1}^{\infty} v_{n}$ is uniformly convergent in $\mathbb{R}$. Let $u(t)=\sum_{n=1}^{\infty} v_{n}$. Clearly, we get

$$
u(t)=\int_{-\infty}^{t} T_{-1}(t-s) f(s) d s, \quad t \in \mathbb{R},
$$

and from Lemma 4.1 we conclude that $u \in \mathrm{AA}\left(X_{0}\right)$.

REMARK 4.3. An immediate consequence of Lemma 4.2 is the existence of almost automorphic mild solution for the nonhomogeneous linear peoblem

$$
u^{\prime}(t)=A u(t)+f(t), \quad t \in \mathbb{R},
$$

where $A$ is a Hille-Yosida operator of negative type on a Banach space $X$ and $f \in \operatorname{AS}^{p}(X) \cap C(\mathbb{R}, X)$, with $p>1$.

Theorem 4.4. Let $f \in \operatorname{AS}^{p}\left(X_{0}, X\right)$ and suppose that there is a constant $L>0$ such that

$$
\|f(t, x)-f(t, y)\| \leq L\|x-y\|, \quad \text { for all } t \in \mathbb{R} \text { and all } x, y \in X_{0} .
$$

Then the equation (1.1) has a unique mild solution $u \in \mathrm{AA}\left(X_{0}\right)$, whenever $M L /|\omega|<1$.

Proof. Define $\Lambda$ on $\mathrm{AA}\left(X_{0}\right)$ by (3.3). Using Lemmas 2.10 and 4.2, we have that $\Lambda\left(\mathrm{AA}\left(X_{0}\right)\right) \subset \mathrm{AA}\left(X_{0}\right) \subset \mathrm{AS}^{p}\left(X_{0}\right)$. Therefore, $\Lambda: \mathrm{AA}\left(X_{0}\right) \rightarrow \mathrm{AA}\left(X_{0}\right)$ is well defined. On the other hand,

$$
\|\Lambda u(t)-\Lambda v(t)\| \leq \int_{-\infty}^{t} M e^{\omega(t-s)} L\|u(s)-v(s)\| d s \leq \frac{M L}{|\omega|}\|u-v\|_{\infty},
$$

for every $t \in \mathbb{R}$. Therefore, the result is consequence of the Banach fixed point theorem.

We close this work with the following example.

EXAmPle 4.5 (Example 3.12 revisited). Let $\Omega \subset \mathbb{R}^{m}$ be a bounded open set with regular boundary $\partial \Omega$. Consider the problem

$$
\begin{cases}u_{t}=\Delta u+a f(u) & \text { in } \mathbb{R} \times \Omega, \\ u=0 & \text { on } \mathbb{R} \times \partial \Omega,\end{cases}
$$

where, $a \in \mathrm{AS}^{p}(\mathbb{R})$ and $f \in C(\mathbb{R}, \mathbb{R})$ is a globally $L$-Lipschitz function. With the same procedure of the Example 3.12 we can rewrite the problem (4.2) in the abstract form (1.1). Then, if $L$ is small enough we have that the problem (4.2) has a unique almost automorphic mild solution. 


\section{REFERENCES}

[1] B. Amir and L. Maniar, Composition of pseudo-almost periodic functions and Cauchy problems with operator of non dense domain, Ann. Math. Blaise Pascal 6 (1) (1999), 1-11.

[2] K. Ammari and G. Vodev, Boundary stabilization of the transmission problem for the Bernoulli-Euler plate equation, Cubo 11 (2009), 39-49.

[3] S. Bochner, Continuous mapping of almost automorphic and almost periodic functions, Proc. Nat. Acad. Sci. USA 52 (1964), 907-910.

[4] S. Boulite, L. Maniar and G. N'GuÉréKata, Almost automorphic solutions for hyperbolic semilinear evolution equations, Semigroup Forum 71 (2) (2005), 231-240.

[5] K.J. Engel AND R. NAGeL, One-parameter semigroups for linear evolution equations, Graduate Texts in Mathematics, Springer-Verlag, 2001, p. 194.

[6] G.A. Goldstein and G.M. N'GuÉRÉKAta, Almost automorphic solutions of semilinear evolution equations, Proc. Amer. Math. Soc. 133 (8) (2005), 2401-2408.

[7] J. Liang, J. Zhang And T.J. XIAO, Composition of pseudo almost automorphic and asymptotically almost automorphic functions, J. Math. Anal. Appl. 340 (2008), 14931499.

[8] J. LiU AND X. Song, Almost automorphic and weighted pseudo almost automorphic solutions of semilinear evolution equations, J. Funct. Anal. 258 (2010), 196-207.

[9] R. NAGel ANd E. SinestraRI, Inhomogeneous Volterra Integrodifferential Equations for Hille-Yosida Operators, Lectures Notes Pure Appl. Math., Marcel Dekker, 1994, p. 150.

[10] G.M. N'GuÉRÉKata, Almost Automorphic and Almost Periodic Functions in Abstract Spaces, Kluwer Acad/Plenum, New York, Boston, Moscow, London, 2001.

[11] G.M. N'GuÉrÉkata And A. Pankov, Stepanov-like almost automorphic functions and monotone evolution equations, Nonlinear Anal. 68 (2008), 2658-2667.

[12] A. PAZY, Semigroups of Linear Operators and Applications to Partial Differential Equations, Spinger, New York, 1983.

[13] G. Da Prato and P. Grisvard, On extrapolation spaces, Atti Accad. Naz. Lincei Rend. Cl. Sci. Fis. Mat. Natur. (8) 72 (1982), no. 6, 330-332.

[14] G. Da Prato and E. Sinestrari, Differential operators with non dense domain, Ann. Scuola Norm. Sup. Pisa Cl. Sci. (4) 14 (1989), no. 2, 285-344.

Bruno de Andrade

Universidade de São Paulo

Instituto de Ciências Matemáticas e de Computação

Departamento de Matemática

CEP. 13569-970, São Carlos-SP, BRAZIL

E-mail address: bruno00luis@gmail.com

Eder Mateus and Arlúcio Viana

Universidade Federal de Sergipe

Campus Professor Alberto Carvalho

Departamento de Matemática-DMAI

CEP. 49500-000, Itabaiana-SE, BRAZIL

E-mail address: edermateus@ufs.com, arlucioviana@ufs.com

TMNA : Volume $44-2014-\mathrm{N}^{\mathrm{O}} 1$ 